\title{
Epidemiological Characteristics of Echinococcosis in Non-Endemic PLADs — China, 2017-2020
}

\author{
Yan Kui ${ }^{1}$; Baixue Liu'; Xu Wang'; Cuizhao Xue'; Jingbo Xue'; Yalan Zhang'; \\ Benguang Zhang; ${ }^{3}$; Yang $\mathrm{Dai}^{4}$; Shuai Han ${ }^{1, *}$; Weiping $\mathrm{Wu}^{1, *}$
}

\section{Summary \\ What is already known about this topic?}

Echinococcosis, also known as hydatid disease, is a zoonotic parasitic disease caused by the larvae of Echinococcus granulosus. Western China has one of the most severe epidemics worldwide. Echinococcosis is endemic in 370 counties of 9 provincial-level administrative divisions (PLADs) including Inner Mongolia, Sichuan, Yunnan, Tibet, Shaanxi, Gansu, Qinghai, Ningxia, Xinjiang and Xinjiang Production and Construction Corps (XPCC).

\section{What is added by this report?}

From 2017 to 2020, 244 cases were reported in 21 non-endemic PLADs. Of the cases reported in nonendemic PLADs, the majority were imported from endemic areas. Cases reported from non-endemic PLADs have been sporadic, and the number has increased in some areas.

What are the implications for public health practice?

Migrant workers and livestock from endemic areas may have contributed to the increased incidence of locally acquired infections in non-endemic PLADs, suggesting that health education among workers and livestock quarantine is important for the control of spread in non-endemic PLADs.

Echinococcosis, also known as hydatid disease, is a zoonotic parasitic disease caused by the larvae of Echinococcus granulosus. Echinococcosis has been hyperendemic in the Qinghai-Tibet Plateau, which has the highest prevalence worldwide (1). In recent years, the prevention and treatment of echinococcosis in China has improved. However, many difficulties and challenges remained (2). This study aimed to analyze the endemic distribution and epidemiological characteristics of reported cases in the non-endemic provincial-level administrative divisions (PLADs) of echinococcosis in China from 2017 to 2020. The regional distribution, age, and gender of reported cases were statistically analyzed, and onsite epidemiological investigations were conducted to clarify the sources of infection of the cases. The results showed that the epidemiological survey revealed the presence of suspected local cases in 15 PLADs, with an increasing yearly trend of reported cases in non-endemic PLADs. Endemic echinococcosis is expanding, and there may be potentially new endemic areas. Echinococcosis endemic surveillance should be strengthened, while the quality of field epidemiological investigations of echinococcosis should be improved to provide a basis for optimizing the existing prevention and control strategies and measures.

In China, echinococcosis is currently classified as a Category $\mathrm{C}$ infectious disease. Cases of echinococcosis have also been reported in non-endemic PLADs and consisted of both suspected local cases and imported cases. In this study, the cases of echinococcosis reported to the National Notifiable Diseases Reporting System (NNDRS) of the China CDC from nonendemic PLADs from 2017 to 2020 were analyzed. Specifically, this study included onsite epidemiological investigations, an assessment of the epidemic status and epidemiological characteristics, and identification of the sources of infections in order to provide a basis for optimizing the prevention and control strategies for echinococcosis. Microsoft Excel (version 2016, Microsoft Corp, Redmond, USA), SAS Software (version 9.4, SAS Institute Inc., NC, USA) were used for basic descriptive statistical analyses. The geographic distribution of cases, age, gender, and source of infection were analyzed using the chi-squared test $(P<0.05)$. Based on the 2012-2016 national echinococcosis epidemiological survey results, 9 PLADs and XPCC were identified as echinococcosis endemic PLADs (3) (Figure 1). Locally-acquired cases refer to no history of living in endemic areas. For cases reported in non-endemic PLADs, investigations and verifications were carried out by staff from national and provincial offices of CDC for the Prevention and 


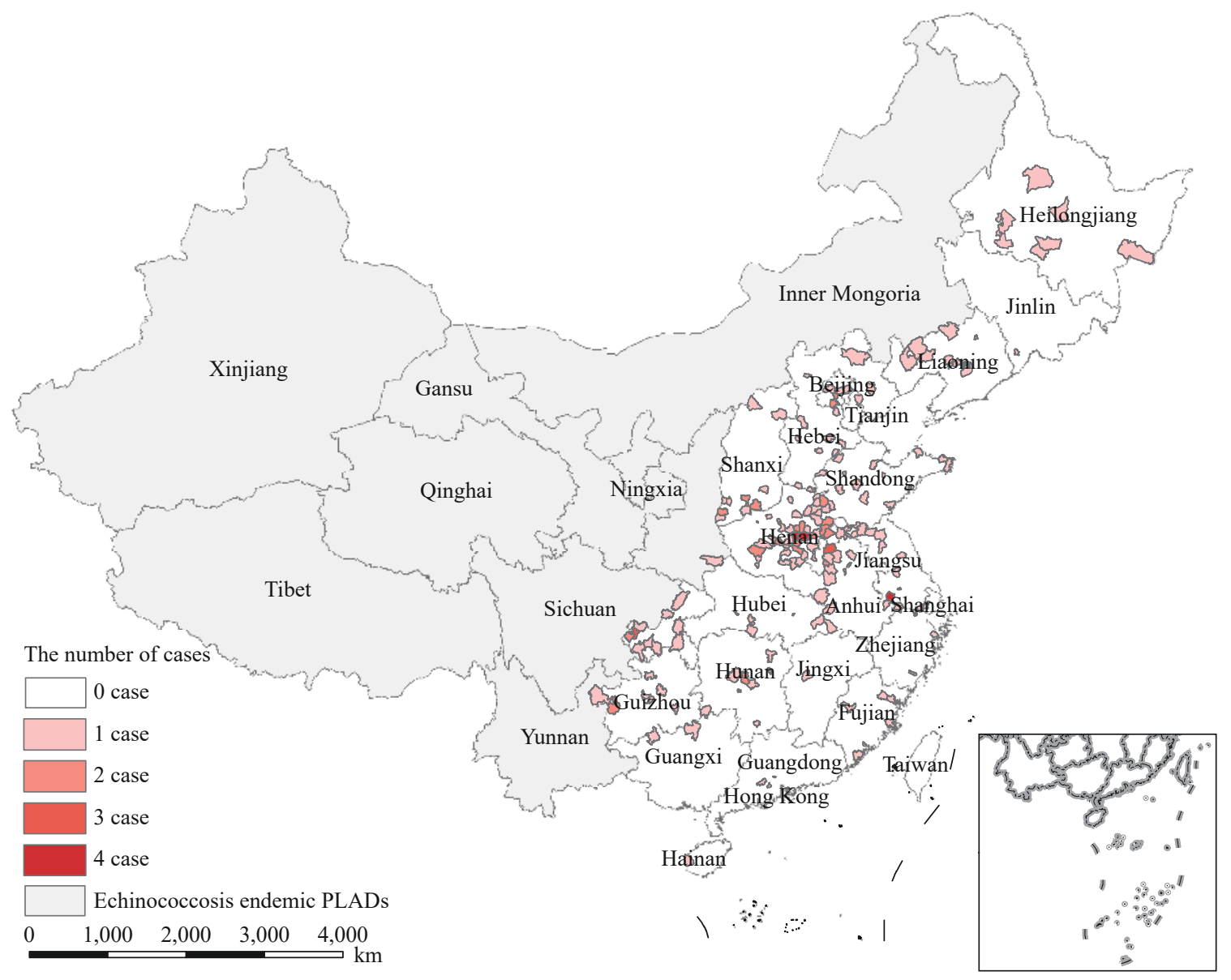

FIGURE 1. The regional distribution of reported cases of echinococcosis in non-endemic counties of PLADs' of China from 2017 to 2020.

Abbreviation: PLADs=provincial-level administrative divisions.

Control of Parasitic Diseases. Reported cases of echinococcosis from 2017 to 2020 were extracted from the China CDC NNDRS and duplicate reports were excluded.

From 2017 to 2020, 19,948 cases of echinococcosis were reported nationwide, of which 19,704 cases were reported in endemic PLADs. Of the cases from endemic areas, the majority $(80.85 \%)$ were reported in Sichuan, Qinghai, and Xinjiang. The remaining 244 cases were reported in 21 non-endemic PLADs (Figure 1) and were mainly distributed in Henan, Shandong, Anhui, Shanxi, Jiangsu, Chongqing, and Hebei, among which Henan Province had the highest number of non-endemic cases $(28.28 \%)$ (Table 1 ). Cases were reported throughout the year with no significant seasonal variation. However, the number of non-endemic reported cases were sporadic and locally increasing. (Figure 1).

The age ranged from 3 to 85 years with a mean age of $44.5 \pm 16.2$ years. The age distribution of cases was as follows $\left(\chi^{2}=52.1211, \quad P<0.05\right):<10$ years $(\mathrm{n}=10$; $4.10 \%), 10-19$ years $(\mathrm{n}=6 ; 2.46 \%), 20-29$ years $(\mathrm{n}=32 ; 13.11 \%), 30-39$ years $(\mathrm{n}=30 ; 12.30 \%), 40-49$ years $(\mathrm{n}=68 ; 27.87 \%), 50-59$ years $(\mathrm{n}=60 ; 24.59 \%)$, and $\geq 60$ years $(n=38 ; 15.57 \%)$. There was no significant difference between men and women [54.1\% and $\left.45.9 \%,\left(\chi^{2}=0.8211, P>0.05\right)\right]$.

The most frequently reported occupational categories were $\left(\chi^{2}=170.3174, \quad P<0.05\right)$ : farmers $(\mathrm{n}=114 ; 46.72 \%)$, domestic and unemployed individuals $(\mathrm{n}=50 ; 20.49 \%)$, retired individuals $(\mathrm{n}=18$; $7.38 \%)$, and others $(n=14 ; 5.74 \%)$. No other occupational category had more than 10 cases.

Of the 244 cases reported in non-endemic PLADs, there were 58 locally-acquired cases $(23.77 \%), 165$ imported cases $(67.62 \%)$, and 21 cases $(8.61 \%)$ that were lost to follow-up. The sources of the imported cases were as follows: $3(1.23 \%)$ from Gansu, 16 $(6.56 \%)$ from Inner Mongolia, $2(0.82 \%)$ from Ningxia, 15 (6.15\%) from Tibet, 109 (44.67\%) from 
TABLE 1. The number of reported cases of echinococcosis in non-endemic PLADs - China, 2017-2020.

\begin{tabular}{|c|c|c|c|c|c|}
\hline PLADs & Cumulative cases $(n)$ & Composition ratio (\%) & $\begin{array}{l}\text { Locally cumulative } \\
\text { acquired cases (n) }\end{array}$ & $\begin{array}{c}\text { Cumulative imported } \\
\text { cases }(n)\end{array}$ & $\begin{array}{c}\text { Cumulative missing } \\
\text { cases }(n)\end{array}$ \\
\hline Beijing & 7 & 2.87 & 0 & 6 & 1 \\
\hline Hebei & 13 & 5.33 & 2 & 10 & 1 \\
\hline Shanxi & 14 & 5.74 & 7 & 6 & 1 \\
\hline Liaoning & 8 & 3.28 & 3 & 3 & 2 \\
\hline Jilin & 1 & 0.41 & 0 & 1 & 0 \\
\hline Heilongjiang & 8 & 3.28 & 1 & 6 & 1 \\
\hline Shanghai & 2 & 0.82 & 0 & 1 & 1 \\
\hline Jiangsu & 14 & 5.74 & 6 & 8 & 0 \\
\hline Zhejiang & 5 & 2.05 & 1 & 4 & 0 \\
\hline Anhui & 18 & 7.38 & 3 & 12 & 3 \\
\hline Fujian & 7 & 2.87 & 6 & 1 & 0 \\
\hline Jiangxi & 1 & 0.41 & 1 & 0 & 0 \\
\hline Shandong & 27 & 11.07 & 8 & 19 & 0 \\
\hline Henan & 69 & 28.28 & 8 & 59 & 2 \\
\hline Hubei & 7 & 2.87 & 0 & 6 & 1 \\
\hline Hunan & 10 & 4.10 & 1 & 7 & 2 \\
\hline Guangdong & 4 & 1.64 & 0 & 4 & 0 \\
\hline Guangxi & 3 & 1.23 & 3 & 0 & 0 \\
\hline Hainan & 1 & 0.41 & 1 & 0 & 0 \\
\hline Chongqing & 14 & 5.74 & 0 & 10 & 4 \\
\hline Guizhou & 11 & 4.51 & 7 & 2 & 2 \\
\hline Total & 244 & 100.00 & 58 & 165 & 21 \\
\hline
\end{tabular}

Abbreviation: PLADs=provincial-level administrative divisions.

Xinjiang, $1(0.41 \%)$ from Yunnan, 7 (2.87\%) from Sichuan, $10(4.10 \%)$ from Qinghai, and $2(0.82 \%)$ from other countries (Figure 2). Furthermore, there were also cases of locally-acquired infections reported in 15 PLADs, imported cases in 18 PLADs, and cases that were lost to follow-up in 11 PLADs (Table 1).

\section{DISCUSSION}

The results of this study showed the dynamic changes and epidemiological characteristics of reported echinococcosis cases in China from 2017 to 2020. There were 19,948 cases of echinococcosis reported nationwide from 2017 to 2020, of which 244 cases were reported in 21 non-endemic PLADs. Henan Province reported the largest number of cases. Cases were distributed across all age groups, with the majority of cases occurring in people aged $>20$ years. Furthermore, the majority of patients were farmers. The epidemiological investigation found that of the cases reported in non-endemic PLADs, the majority were imported and less than one-quarter were confirmed to have been locally acquired. The largest source of imported cases was Xinjiang. There were locally acquired cases reported in 11 non-endemic PLADs. According to the China Statistical Yearbook (4), from 2000 to 2019, the migrant population in China increased from 121 million to 236 million, the number of domestic tourists increased from 744 million to 6.006 billion, meat production increased from 60.139 million tons to 77.588 million tons, and the total output value of the livestock industry grew from 739.31 billion CNY to 3,306.43 billion CNY. The increase in population migration (due to work, tourism, and labor) and the circulation of livestock and other products between regions could pave the way for the spread of echinococcosis from pasturing areas to agricultural and urban areas.

This increase in the number of cases reported in non-endemic PLADs has been reported previously (5), and it is expected that this trend will continue. This increase is likely to be attributable to the gradual 


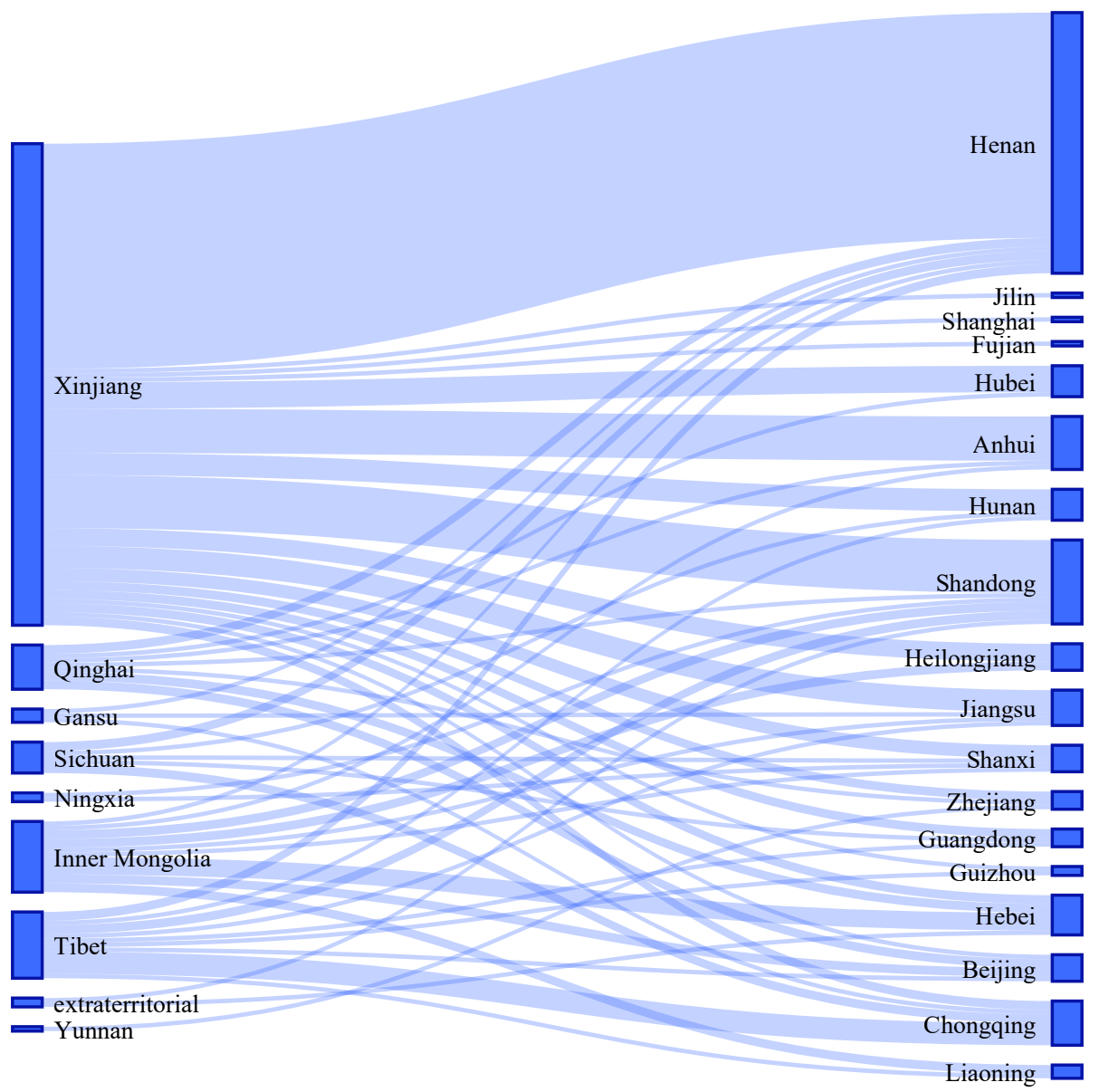

FIGURE 2. The Sankey diagram of exported PLADs and imported non-endemic PLADs of echinococcosis in China from 2017 to 2020.

Abbreviation: PLADs=provincial-level administrative divisions.

increase in the detection of echinococcosis through screening and improved health-seeking behavior. Moreover, hospitals and county-level CDCs have strengthened the awareness of reporting notifiable diseases and cases. According to the results of the epidemiological investigation of suspected local cases, no infected dogs were found, and the cases were sporadic. However, some local livestock originated from endemic areas. It is speculated that the humans were infected by ingesting insect eggs on animal fur or by people feeding diseased organs to dogs in nonendemic areas which led to the spread of echinococcosis. Infected individuals in non-endemic PLADs were mainly those of working age, the possibility of infection when they were working in endemic areas cannot be ruled out. This suggests that health education for people working in endemic areas should be strengthened. Some of the locally acquired cases in non-endemic PLADs may have been related to work, such as fur processing. Most cases from non- endemic PLADs were reported from Henan, Heilongjiang, and Jiangsu (O), which suggests that echinococcosis may also be endemic in parts of China where it is not currently recognized as endemic and there is still a risk of locally acquired infections. More detailed onsite investigation needs to be the next step, including mass screening of high-risk population and surveys of intermediate and final. The National Echinococcosis Control Expert Group should determine if there are new endemic areas based on the field investigation results.

The study was subject to some limitations. Because echinococcosis is a chronic disease (7), of which there are two types including cystic echinococcosis (CE) and alveolar echinococcosis (AE), the progression of the disease is hard to classify due to different imaging features $(8-10)$. The NNDRS did not specify whether these cases were $\mathrm{CE}$ or $\mathrm{AE}$, so the prevalence of echinococcosis in China may not be fully reflected by the number of cases as some may be misclassified or 
missed due to limitations in the surveillance system.

doi: $10.46234 / \mathrm{ccdcw} 2021.262$

\# Corresponding authors: Shuai Han, hanshuai@nipd.chinacdc.cn; Weiping Wu,wuwp@nipd.chinacdc.cn.

\begin{abstract}
${ }^{1}$ National Institute of Parasitic Diseases, Chinese Center for Disease Control and Prevention (Chinese Center for Tropical Diseases Research); National Health Commission Key Laboratory of Parasite and Vector Biology; World Health Organization Collaborating Center for Tropical Diseases; National Center for International Research on Tropical Diseases, Shanghai, China; ${ }^{2}$ Henan Provincial Center for Disease Control and Prevention, Zhengzhou, Henan, China; ${ }^{3}$ Shandong Institute of Parasitic Diseases, Jining, Shandong, China;

${ }^{4}$ Jiangsu Institute of Parasitic Diseases, Wuxi, Jiangsu, China.
\end{abstract}

Submitted: July 19, 2021; Accepted: December 14, 2021

\section{REFERENCES}

1. Wu WP, Wang H, Wang Q, Zhou XN, Wang LY, Zheng CJ, et al. A nationwide sampling survey on echinococcosis in China during 20122016. Chin J Parasitol Parasitic Dis 2018;36(1):1-14. http://www. jsczz.cn/CN/Y2018/V36/I1/1. (In Chinese).

2. Fu MH, Han S, Xue CZ, Wang X, Liu BX, Wang Y, et al. Contribution to the echinococcosis control programme in China by NIPD-CTDR. Adv Parasitol 2020;110:107 - 44. http://dx.doi.org/10. 1016/bs.apar.2020.04.010

3. National Health and Family Planning Commission, Central United Front Work Department, National Development and Reform Commission, Ministry of Education, Ministry of Science and Technology, Ministry of Public Security, et al. National Program for the Prevention and Control of Key Parasitic Diseases including Hydatidosis (2016-2020). National Health and Family Planning Commission Disease Control Bureau letter [2016] no. 58. http://www. nhc.gov.cn/jkj/s5873/201702/dda5ffe3f50941a29fb0aba6233bb497. shtml. [2016-10-24]. (In Chinese).

4. National Bureau of Statistics. China statistical yearbook 2020. Beijing: China Statistics Press, 2020. http://www.stats.gov.cn/tjsj/ndsj/2020/ indexch.htm. (In Chinese).

5. Han S, Wu WP, Xue CZ. Analysis of echinococcosis cases from nonendemic areas of China in 2017. J Parasitic Biol 2019;14(8):901-4,909. http://www.cjpb.org.cn/artshow.asp?id=8769. (In Chinese).

6. Huang XM, Huang LZ, Lü LF, Jiang L, Xie YQ, Xu XZ. Epidemiological survey on echinococcosis prevalence in Liyang City of Jiangsu Province from 2013 to 2018. J Trop Dis Parasitol 2019;17(3):153-5. http://www.rdbzz.com/CN/abstract/abstract556. shtml. (In Chinese).

7. Wang Q, Huang Y, Huang L, Yu WJ, He W, Zhong B, et al. Review of risk factors for human echinococcosis prevalence on the Qinghai-Tibet Plateau, China: a prospective for control options. Infect Dis Poverty 2014;3:3. http://dx.doi.org/10.1186/2049-9957-3-3.1.

8. Wang Q, Vuitton DA, Qiu JM, Giraudoux P, Xiao YF, Schantz PM, et al. Fenced pasture: a possible risk factor for human alveolar echinococcosis in Tibetan pastoralist communities of Sichuan, China. Acta Trop 2004;90(3):285 - 3. http://dx.doi.org/10.1016/j.actatropica. 2004.02.004.

9. WHO Informal Working Group. International classification of ultrasound images in cystic echinococcosis for application in clinical and field epidemiological settings. Acta Trop 2003;85(2):253-61. http:/ /dx.doi.org/10.1016/s0001-706x(02)00223-1.

10. Chinese Doctor Association, Chinese College of Surgeons (CCS), Chinese Committee for Hadytidology (CCH). Expert consensus on diagnosis and treatment of hepatic cystic and alveolar echinococcosis (2019 edition). Chin J Dig Surg 2019;18(8):711 - 21. http://dx.doi. org/10.3760/cma.j.issn.1673-9752.2019.08.002. (In Chinese). 